\title{
Biexciton spin and angular momentum transitions in vertically coupled quantum dots
}

\author{
Egidijus Anisimovas ${ }^{1,2, *}$ and F. M. Peeters ${ }^{1, \dagger}$ \\ ${ }^{1}$ Departement Fysica, Universiteit Antwerpen (Campus Drie Eiken), B-2610 Antwerpen, Belgium \\ ${ }^{2}$ Semiconductor Physics Institute, Goštauto 11, LT-01108 Vilnius, Lithuania
}

(Received 19 November 2004; published 22 March 2005)

\begin{abstract}
An "exact" numerical calculation is presented for the energy spectrum of biexcitons, with spatially separated electrons and holes, in coupled quantum dots in the presence of a perpendicular magnetic field. When the interdot distance is small the two electrons and two holes form a strongly correlated complex whose spin state undergoes characteristic transitions with increasing interdot separation and magnetic field. For the smallest distances and magnetic fields the biexciton is bound with respect to dissociation into two excitons. At larger distances we observe angular momentum transitions with sawtooth-shaped phase boundaries. The biexciton diamagnetic shift shows a sign reversal as a function of the interdot distance.
\end{abstract}

DOI: $10.1103 /$ PhysRevB.71.115319

PACS number(s): 73.21.La, 78.67.Hc

\section{INTRODUCTION}

Ground-state angular momentum transitions define a particularly relevant topic and a point of interest in physics of few-electron quantum dots. ${ }^{1-4}$ With increasing magnetic field the extent of the electron wave function in the quantum dot is reduced and thus increases the Coulomb interaction energy up to the point when it becomes energetically more favorable for the electrons to jump to higher angular momentum orbitals, thus increasing the interparticle separation and consequently reducing the Coulomb repulsion. These transitions frequently go hand-in-hand with transitions of the spin multiplicity, ${ }^{5-8}$ since only certain combinations of the orbital and spin quantum numbers (usually referred to as "magic numbers") are compatible. While the increase of the angular momentum with increasing external magnetic field strength is a classical phenomenon, ${ }^{1,9}$ the quantization of the angular momentum leads to abrupt changes in the quantum dot size and various contributions to the total energy of the quantum dot. ${ }^{1}$ A recent experiment ${ }^{10}$ succeeded in detecting the dot area changes by around $10 \%$ in a capacitance spectroscopy measurement. The transitions also induce oscillations of other observable quantities such as the electronic heat capacity $^{7,11}$ and magnetization. ${ }^{5,6}$

The addition of holes, which are positively charged and thus introduce attractive forces into the system, adds new physics to the quantum dot problem. In vertically coupled quantum dots and double quantum-well ${ }^{12}$ systems electrons and holes can be spatially separated (e.g., through an internal or externally applied electric field) and thus have much larger radiative lifetimes. The interplay between the Wigner crystallization and the formation of interdot excitons in vertically coupled dots was recently investigated using path integral Monte Carlo simulations. ${ }^{13}$ Four distinct states of the electron-hole system were identified as both excitonic, and decoupled electron and hole phases can form in either a liquid or a crystal state. The magnetoplasma oscillations in a double-dot electron-hole system were also studied $^{14}$ and a strong coupling between the center-of-mass modes of the individual electron and hole systems was found. Effects induced by the interdot tunneling in double quantum dots have been considered, ${ }^{15,16}$ and unexpected spa- tial correlations between the carriers were discovered. In particular, it was shown that the exciton tunneling dominates over the individual tunneling of electrons and holes, and localization of two electron-hole pairs in one dot may occur. ${ }^{16}$ The most interesting phenomenon to be looked for in these systems is the predicted Bose-Einstein condensation of the interlayer excitons. ${ }^{17-19}$

In a previous paper $^{20}$ we considered the ground-state angular momentum transitions of a negatively charged exciton confined in a double quantum dot. It was found that the presence of a hole modifies the transitions in the electronic subsystem by partially screening the electron-electron interaction. However, the hole subsystem itself (consisting in this case of only one particle) remains largely inert. The charge redistributions change the radius of the hole dot by only $1 \%-2 \%$. The purpose of the present paper is to study a biexciton, i.e., a complex of two electrons and two holes, where we will concentrate on the ground-state phase diagram, the possibility for the biexciton to become bound, and the diamagnetic shift.

The paper is organized as follows. Section II describes the model used and the numerical procedure. Sections III and IV present the results obtained at small interlayer separations: spin multiplicity transitions and binding of the biexcitons. In Sec. V angular momentum transitions at larger separations are considered, and Sec. VI discusses the diamagnetic shifts. We summarize our results in Sec. VII.

\section{MODEL}

Our model involves two strictly two-dimensional electrons of effective mass $m^{*}$ and charge $-e$ moving in one layer, and two holes of mass $M^{*}$ and charge $+e$ moving in a parallel layer separated by a vertical distance $\mathbf{d}$. The lateral confinement in the layers is, in both cases, assumed to be circularly symmetric and parabolic with the confining frequencies $\omega_{0}$ and $\Omega_{0}$ for electrons and holes, respectively. The two oscillator radii are given by

$$
l_{0}=\sqrt{\frac{\hbar}{m^{*} \omega_{0}}} \quad \text { and } L_{0}=\sqrt{\frac{\hbar}{M^{*} \Omega_{0}}},
$$

and we set these lengths to $l_{0}=2.5 a_{B}^{*}$ and $L_{0}=2 a_{B}^{*}$ 
$\left(a_{B}^{*}=\epsilon \hbar^{2} / m^{*} e^{2}\right.$ is the effective electron Bohr radius), thus accounting for the fact that the holes are typically more strongly confined. The magnetic field is described in terms of the symmetric-gauge vector potential $\mathbf{A}=\frac{1}{2}[\mathbf{B} \times \mathbf{r}]$.

The Hamiltonian of the system consists of single-body, Zeeman, and Coulomb interaction parts,

$$
H=\sum_{i=1}^{2} T_{e i}+\sum_{i=1}^{2} T_{h i}+H_{Z}+V_{C} .
$$

The single-particle energies of electrons and holes, respectively, are given by

$$
\begin{aligned}
T_{e i} & =\frac{\hbar^{2}}{2 m^{*}}\left[\mathbf{p}_{e i}+\frac{e}{c} \mathbf{A}\left(\mathbf{r}_{e i}\right)\right]^{2}+\frac{1}{2} m^{*} \omega_{0}^{2} r_{e i}^{2}, \\
T_{h i} & =\frac{\hbar^{2}}{2 M^{*}}\left[\mathbf{p}_{h i}-\frac{e}{c} \mathbf{A}\left(\mathbf{r}_{h i}\right)\right]^{2}+\frac{1}{2} M^{*} \Omega_{0}^{2} r_{h i}^{2},
\end{aligned}
$$

and the Zeeman Hamiltonian is

$$
H_{Z}=\mu_{B} B\left(g_{e}^{*} S_{e z}+g_{h}^{*} S_{h z}\right),
$$

where $\mu_{B}$ is the Bohr magneton, $g_{e, h}^{*}$ are the effective electron and hole gyromagnetic factors, and $S_{e z, h z}$ are the total spin projections of electrons and holes.

The interaction part $V_{C}$ is the sum of the Coulomb interaction terms between all pairs of particles. When the two involved particles are of the same kind and, accordingly, move in the same layer the respective term reads

$$
V_{i j}=\frac{e^{2}}{\epsilon} \frac{1}{\left|\mathbf{r}_{i}-\mathbf{r}_{j}\right|},
$$

while for different particles this term becomes

$$
V_{i j}=-\frac{e^{2}}{\epsilon} \frac{1}{\left|\mathbf{r}_{i}-\mathbf{r}_{j}+\mathbf{d}\right|},
$$

with $\mathbf{d}$ being the vertical distance between the layers. Since the dots are vertically separated, the overlap between the electron and hole wave functions is exponentially small and thus we may neglect the electron-hole exchange interaction.

We work in dimensionless "atomic" units set by the electron material parameters, i.e., $m^{*}=e^{2} / \epsilon=\hbar=1$. Thus, the lengths are measured in the electron Bohr radii $a_{B}^{*}=\epsilon \hbar^{2} / m^{*} e^{2}$ and the energies in the electron effective Hartree energies $E_{H}^{*}=e^{2} / \epsilon a_{B}^{*}$. If GaAs parameters $\left(\mathrm{m}^{*} / \mathrm{m}_{e}=0.067\right.$ and $\left.\epsilon=12.4\right)$ are assumed, the above units become $a_{B}^{*}=9.8 \mathrm{~nm}$ and $E_{H}^{*}=11.86 \mathrm{meV}$. For the effective hole mass we take the value $M^{*} / m_{e}=0.45$.

Both the electrons and the holes can form either spinsinglet or spin-triplet states, and we label the terms by indicating the spin state as a pair of two numbers ("1" for singlet and " 3 " for triplet), the first pertaining to electrons and the second one to holes. For example, the state in which the electrons form a spin triplet and holes are in a spin-singlet state is denoted by " 31 ". We found it instructive to particularly consider the case without the Zeeman interaction (obtained by setting the effective gyromagnetic factors $\left.g_{e}^{*}=g_{h}^{*}=0\right)$. This limit helps elucidate a systematic pattern of ground-state transitions. However, in GaAs whose material parameters we use, the gyromagnetic factors are not negligible $\left(g_{e}^{*}=-0.44, g_{h}^{*}=0.7\right)$, we also present the results pertaining to this more realistic case.

The eigenvalues and eigenstates of the Hamiltonian (2) are calculated using exact diagonalizations. The basis of many-particle configurations is built of the single-electron and single-hole states in the presence of a parabolic potential and a magnetic field but in the absence of interparticle interactions, i.e., so-called Fock-Darwin states. For electrons, the wave function corresponding to the angular momentum $m$ and the radial quantum number $n$ reads

$$
\varphi_{e}(n m \mid r \theta)=\frac{e^{\operatorname{Im} \theta}}{\sqrt{\pi}} \sqrt{\frac{n !}{(n+|m|) !}} e^{-r^{2} / 2 l^{2}} \frac{r^{|m|}}{l^{|m|+1}} L_{n}^{|m|}\left(\frac{r^{2}}{l^{2}}\right) .
$$

Here, $L_{n}^{k}(x)$ is the associated Laguerre polynomial, and $l$ stands for the magnetic-field-renormalized oscillator length. It is computed from $l^{-4}=l_{0}^{-4}+\frac{1}{4} l_{c}^{-4}$ with $l_{c}=(\hbar c / e B)^{1 / 2}$ being the magnetic length. The expression for the hole states is identical to that given in Eq. (7) with the replacement of the characteristic length $l \rightarrow L$, with $L^{-4}=L_{0}^{-4}+\frac{1}{4} l_{c}^{-4}$. Note that the magnetic length $l_{c}$ is independent of the material parameters. Therefore, although we assume a stronger lateral confinement for the holes, at high magnetic fields the extension of the electron and hole wave functions will approach each other due to the magnetic localization.

In the above-introduced basis the single-particle part of the Hamiltonian is immediately diagonal. The single-electron and single-hole energies are given by

$$
E_{e}(n m)=\hbar \sqrt{\omega_{0}^{2}+\frac{\omega_{c}^{2}}{4}}(2 n+|m|+1)+\frac{1}{2} m \hbar \omega_{c},
$$

$$
E_{h}(n m)=\hbar \sqrt{\Omega_{0}^{2}+\frac{\Omega_{c}^{2}}{4}}(2 n+|m|+1)-\frac{1}{2} m \hbar \Omega_{c},
$$

with $\omega_{c}=e B / m^{*} c$ and $\Omega_{c}=e B / M^{*} c$ denoting the respective cyclotron frequencies. Equation (8) indicates that in strong magnetic fields electrons will preferentially occupy orbitals of negative angular momenta while holes will favor positive ones. The matrix elements of the Coulomb interaction are calculated to a large extent analytically as described in Ref. 20.

Biexciton energies calculated as a function of the applied magnetic field include a background of the single-particle cyclotron energies which may be much larger than the relevant energies determining the transitions between various states. Therefore, we typically calculate and plot the difference of the biexciton energy and the ground-state energy of its constituent particles,

$$
E_{C}\left(X_{2}\right)=E\left(X_{2}\right)-2 E(e)-2 E(h),
$$

which is referred to as the biexciton correlation energy.

One of the relevant issues is whether the biexciton is bound with respect to dissociation into two noninteracting (however, still confined in a quantum-dot molecule) excitons. The biexciton binding energy is defined as the difference between the energy of two excitons and a biexciton so that 

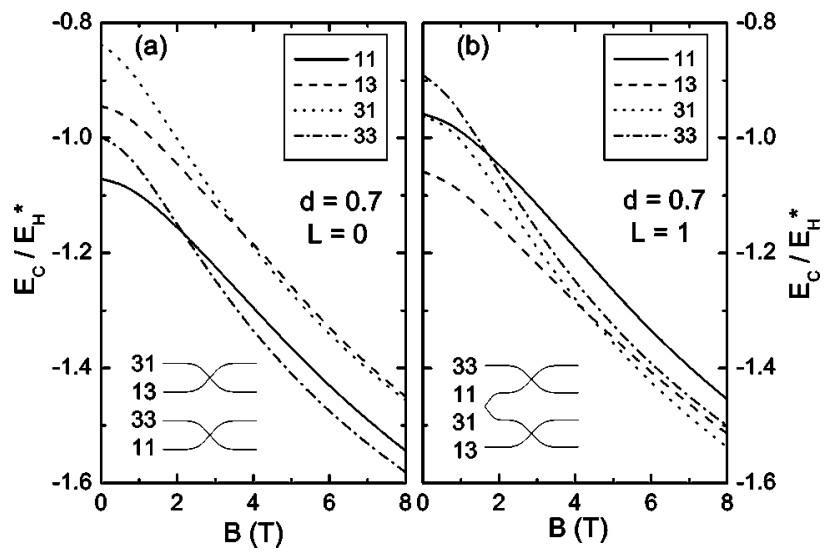

FIG. 1. Magnetic-field evolution of four possible spin configurations in the subspaces of the total angular momentum $L=0$ (a) and $L=1$ (b) in the absence of the Zeeman interaction $\left(g_{e}^{*}=g_{h}^{*}=0\right)$. The different states are identified by a pair of numbers denoting singlet ("1") and triplet ("3") states of, respectively, electrons and holes. The inset shows the schemes of level rearrangement. The ground-state configuration switches from 11 to 33 at around $2.2 \mathrm{~T}$ while in the subspace $L=1$ there is a transition from 13 to 31 at $4.3 \mathrm{~T}$.

its positive value corresponds to bound biexcitons. We express this energy as

$$
E_{B}\left(X_{2}\right)=2 E(X)-E\left(X_{2}\right)=2 E_{C}(X)-E_{C}\left(X_{2}\right),
$$

with the exciton correlation energy $E_{C}(X)=E(X)-E(e)$ $-E(h)$ defined in analogy with Eq. (9).

\section{SINGLET-TRIPLET TRANSITIONS}

We begin by discussing closely spaced dots, in which case the two electrons and two holes form a strongly correlated four-particle complex. Consequently, due to efficient screening of the electron-electron interaction by holes and vice versa, in this limit the tendency to minimize the interaction energy by switching to high-angular-momentum states is suppressed and the ground-state total angular momentum of the quantum dot molecule is $L=0$. However, we still find some interesting transitions of the ground-state spin multiplicity. The nature of these transitions depends on the strength of the Zeeman interaction. For the sake of clarity we first consider the case without the Zeeman term to help understand the systematics of the transitions. Later we explain the role of a nonvanishing Zeeman term which would be present in most realistic cases. In Fig. 1(a), we plot the magnetic field dependence of the correlation energy $E_{C}$ corresponding to four possible biexciton spin configurations when $g_{e}^{*}=g_{h}^{*}=0$, the total angular momentum of the system is $L=0$ and the interdot distance is set to $d=0.7 a_{B}^{*}$. As discussed above, the spin configurations are labeled by the twodigit numbers 11, 13, 31, and 33. One notes in Fig. 1(a) that the different terms cross and rearrange in the way schematically depicted in the inset. The pairs of terms 11 and 33 on one hand and 13 and 31 on the other hand cross each other once in the covered range of magnetic fields up to $8 \mathrm{~T}$. In

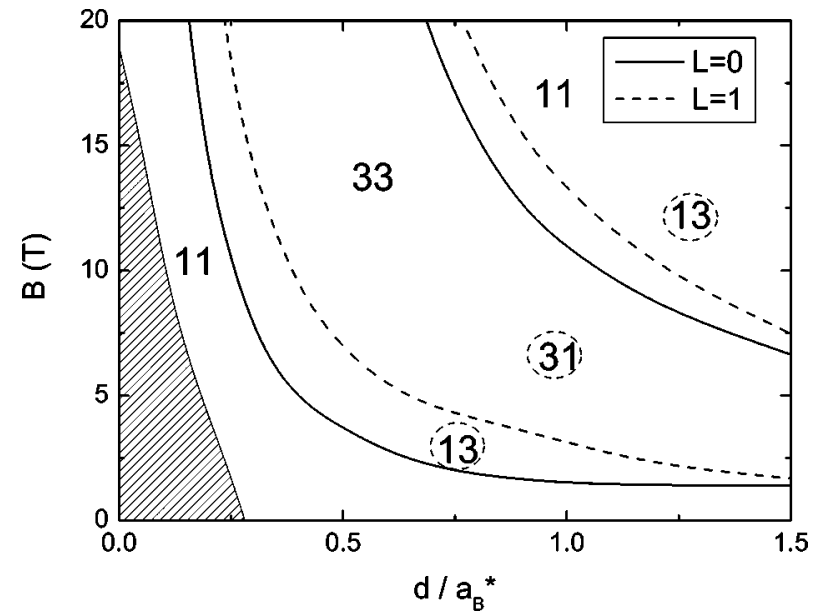

FIG. 2. Phase diagram of biexciton spin-multiplicity transitions in the absence of the Zeeman term. The full lines correspond to the subspace $L=0$, and these transitions are the actual ground-state transitions. With increasing magnetic field and/or interdot distance, at the lower boundary the configuration switches from 11 to 33 and switches back to 11 at the higher boundary. The dotted lines depict the transitions from state 13 to 31 and vice versa in the $L=1$ subspace. The hash-marked area close to the origin indicates the parameter range where the biexciton binding energy is positive.

particular, the spin configuration of the ground state changes from a double singlet to a double triplet at a magnetic field around $2.2 \mathrm{~T}$. A similar transition was found ${ }^{21}$ in an analytical model of tunnel-coupled quantum dots. At higher magnetic fields, the two terms 11 and 33 converge very close to each other in energy, and a transition back to 11 ground state occurs only at a magnetic field of $19 \mathrm{~T}$.

We compare this situation to that observed in the subspace characterized by the total angular momentum $L=1$ (which is not the ground state in the considered parameter range) plotted in Fig. 1(b). One sees that the magnetic field behavior is similar, with the difference that the two lowest terms are 13 and 31 , and the transition is shifted towards higher magnetic fields and takes place at $4.3 \mathrm{~T}$. At even higher magnetic fields the two terms converge towards each other and there is an inconspicuous reentrant transition from 31 to 13 at a magnetic field of $23 \mathrm{~T}$ (not shown in the figure). A qualitatively similar behavior is observed also for higher angular momenta $L$ and for different interdot distances.

These singlet-triplet transitions are summarized in Fig. 2, where we plot the critical magnetic fields at which the spin transitions take place as a function of the separation between the two dots. The full (dotted) lines correspond to the switchings of the spin multiplicity of the lowest energy state in the $L=0(L=1)$ subspaces.

The above-described behavior of energy levels in closely spaced dots can be explained as a modification of the usual singlet-triplet transitions taking place in two-electron (or two-hole) quantum dots. It is well known that in two-particle dots in the absence of the Zeeman interaction the absolute value of the ground-state angular momentum is growing with increasing magnetic field in unit steps, and the even momentum states are spin singlets while the odd momentum states are spin triplets. 

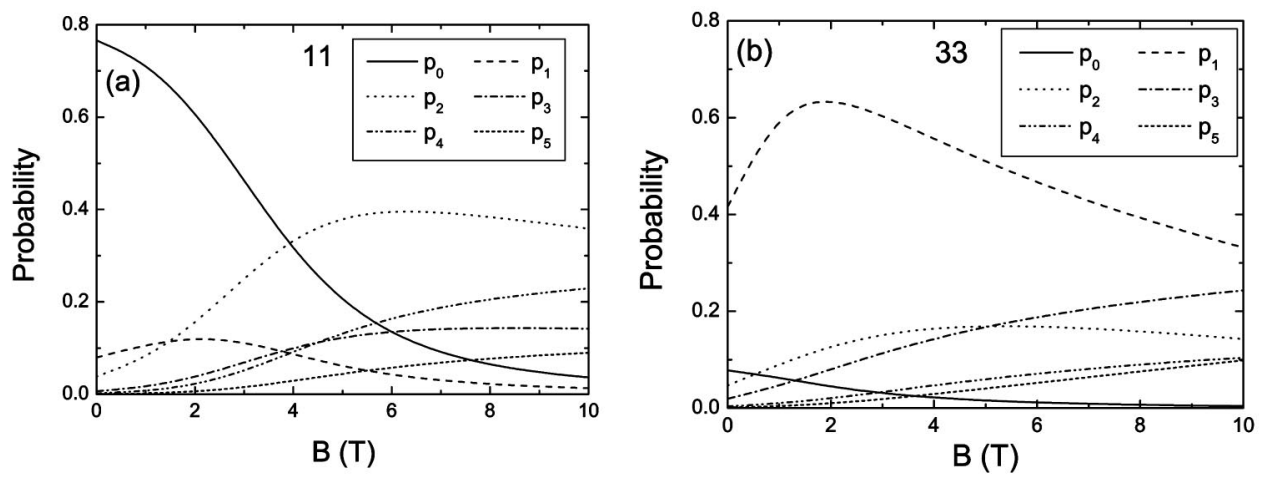

FIG. 3. The probabilities $p_{m}$ to find the holes in a state of total angular momentum $m=0, \ldots, 5$ for the biexciton state $L=0$. The electrons are found in states of total angular momentum $-m$ with the same probabilities. (a) corresponds to the spin configuration 11, and (b) is plotted for the configuration 33. Note that only at the lowest magnetic fields a predominant value of angular momentum can be identified. The interdot distance is $d=0.7 a_{B}^{*}$.

In the present case of two closely spaced dots the angular momentum of each individual dot is not a good quantum number. However, it is still possible to define a most probable value of the angular momentum of an individual dot or its expectation value. In Fig. 3 we plot the probabilities $p_{m}$ to find the hole subsystem in a state of angular momentum $m$. Here we confine our attention to the state with total angular momentum $L=0$, therefore, the distribution of electronic angular momenta will be identical up to a sign. That is, the electron subsystem is found in states of total angular momentum $-m$ with the same probabilities $p_{m}$. The panels 3(a) and 3(b) correspond to the terms 11 and 33, respectively.

One sees that the individual-dot angular momentum values are rather ill-determined, especially at high magnetic fields, so that a dot may be found in many different angular states with appreciable probabilities. Nevertheless, at low magnetic fields the quantum dot is predominantly found in the state $m=0$ for the singlet spin configuration [Fig. 3(a)] and $m=1$ for the spin triplet [Fig. 3(b)]. Moreover, the evenodd effect known for single dots is still quite discernible. It is seen in Fig. 3 that even (odd) momenta are generally preferred over odd (even) momenta in the singlet (triplet) state.

These observations explain the reentrant singlet-triplet transition presented in Fig. 1(a). At zero magnetic field the coupled quantum dots are found in the most probable configuration $m_{e}=0, m_{h}=0$ which favors the spin parts to combine into singlets. At somewhat higher magnetic fields, the preferred state of quantum dots switches into $m_{e}=-1$, $m_{h}=1$, and the spin parts change into triplets. However, after this transition the indeterminacy of individual-dot angular momenta becomes too high, and it is no longer possible to identify transitions into configurations with higher absolute values of $m_{e}$ and $m_{h}$. Therefore, the energies of the spectral terms 11 and 33 become very similar. For the considered interdot distance of $d=0.7$ there still is a transition back to the 11 ground state at $B=19 \mathrm{~T}$, however, for smaller distances such a transition is not found up to very high magnetic fields (see Fig. 2).

The analysis of the singlet-triplet transition in the subspace $L=1$ can be carried out in a very similar fashion and we do not repeat it here. In that case, since the total angular momentum is odd, at a low magnetic field the individual quantum dots will have most probable angular momenta of opposite parity, and consequently, the competition for the lowest energy term will be taking place between the spin configurations 13 and 31. We see from Fig. 2 that the general trend of phase boundaries is very similar to that corresponding to the $L=0$ states.

The inclusion of the Zeeman term with a finite $g_{e, h}^{*}$ does not alter the diagonalization of the Hamiltonian matrix and the above analysis of distribution of particles among various orbital states. It merely resolves the degeneracy of the triplet states and shifts the energy of the lowermost triplet component downwards by an amount proportional to the magnetic field strength thus making this component a more likely candidate for the ground state. In Fig. 4 we plot the energy spectra obtained by setting $g_{e}^{*}=-0.44$ and $g_{h}^{*}=0.7$. In the subspace $L=0$ we still observe the transition from 11 to 33 , which is now shifted to slightly lower magnetic fields $(B=1.6 \mathrm{~T})$. However, at higher magnetic fields the energy of the double-triplet state (33) is further lowered by the Zeeman interaction thus precluding the transition back to the 11 state. In the subspace $L=1$, notable modifications are also seen. The previously found transition $13 \rightarrow 31$ is now revoked since the Zeeman energy of the holes is much higher than that of electrons thus favoring the configuration 13. At higher

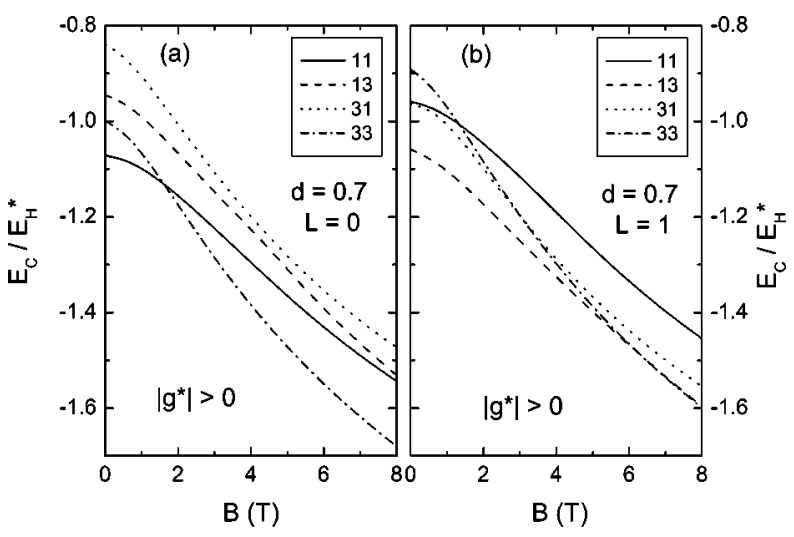

FIG. 4. The same as in Fig. 1 but with the effects of the Zeeman interaction. The most conspicuous transition $11 \rightarrow 33$ survives and is slightly shifted towards lower fields. 


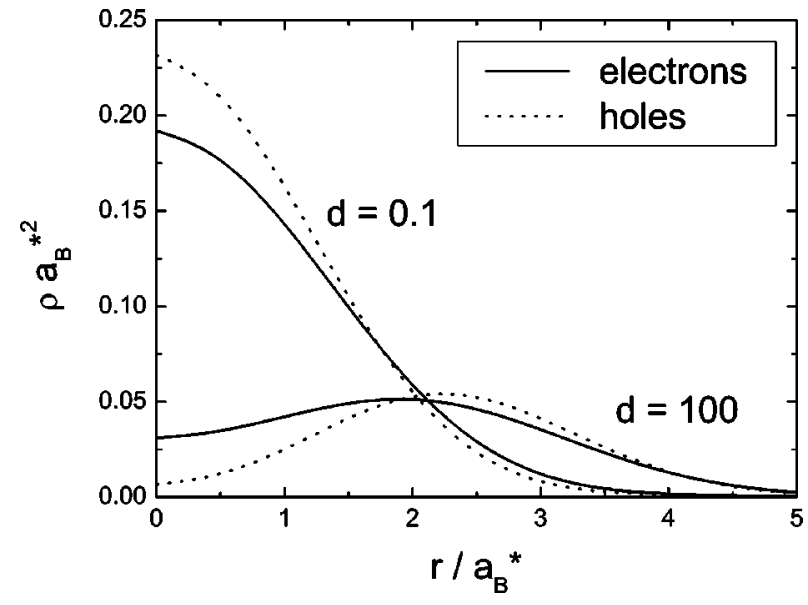

FIG. 5. Electron (full line) and hole (dashed line) charge density distribution in the bound ground state at the interdot separation $d=0.1 a_{B}^{*}$ and magnetic field $B=3 \mathrm{~T}$. Stronger lateral binding of the holes is apparent. For comparison, we show also the charge distribution in decoupled $\left(d=100 a_{B}^{*}\right)$ dots at the same magnetic field.

magnetic fields $(B=6.4 \mathrm{~T})$ the lowest-energy state switches to the configuration 33 where both types of particles are spin polarized.

\section{BOUND BIEXCITONS}

The hash-marked area in Fig. 2 signifies the parameter range where the biexcitons are bound. We see that this binding occurs only at the very lowest values of interdot distance and magnetic field. With increasing $d$ the biexcitons become like two repelling dipoles. Because of the electron and hole confinement potentials the electrons and holes do not move to infinity in the "unbound" region.

In order to see the difference between "bound" and "unbound" biexcitons we plot in Fig. 5 the radial charge distributions of electrons and holes in the bound ground state at $d=0.1 a_{B}^{*}$ and $B=3 \mathrm{~T}$. We see that, due to the strong mutual attraction, the electrons and the holes are confined to a small region close to the center of the dot. Stronger external confinement of the holes results in their narrower distribution. For comparison, we present also the electron and hole charge density distribution obtained at the same magnetic field strength but for decoupled dots $\left(d=100 a_{B}^{*}\right)$. In this case, both the holes and the electrons form ringlike structures since the magnetic field forces them into orbitals of high angular momentum. This effect is stronger for the holes due to their closer spacing of the energy levels.

The correlation between electrons and holes is visualized in Figs. 6(a)-6(d) which show the conditional-probability distribution of electrons corresponding to the same bound ground state at a magnetic field of $B=3 T$ and $d=0.1 a_{B}^{*}$. The two holes are fixed on the $x$ axis opposite to each other at distances $0.5 a_{B}^{*}, a_{B}^{*}, 1.5 a_{B}^{*}, 2.5 a_{B}^{*}$, respectively, from the dot center. One observes that the electrons tend to concentrate close to the dot center, and the position of holes only weakly polarize the almost circular distribution. However, for the largest separation of the holes in Fig. 6(d) it is possible to

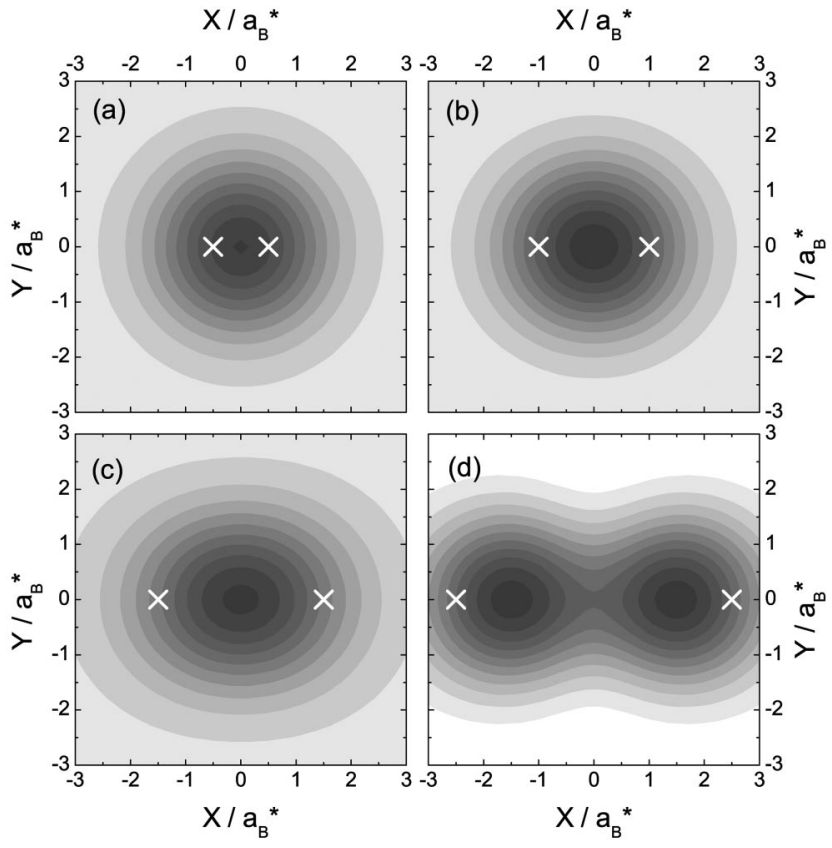

FIG. 6. Electron-hole correlation function-the conditional probability to find an electron when the two holes are fixed opposite to each other at a distance (a) $0.5 a_{B}^{*}$, (b) $a_{B}^{*}$, (c) $1.5 a_{B}^{*}$, and (d) $2.5 a_{B}^{*}$ from the center. The positions of holes are marked with white crosses.

observe a two-peak structure. Note that in this case the holes are situated at the edge of the quantum dot.

\section{ANGULAR MOMENTUM TRANSITIONS}

When the separation between the dots is increased to distances comparable to or larger than the radii of the dots the screening of the interelectron interactions mediated by the nearby holes and vice versa is diminished. Thus, the dot populated by electrons and the dot populated by holes can manifest transitions to higher angular momentum states in an uncorrelated fashion, and a number of angular momentum transitions appear. Here we keep track only of the total angular momentum quantum number and do not identify the different possible spin configurations. In this regime the energies of various spin states converge very closely to each other and are hardly discernible. In Fig. 7 we plot the magnetic field versus the interdot separation-phase diagram corresponding to this regime. One notes that with increasing magnetic field and/or interdot distance the total angular momentum follows a sequence of growing positive values. However, this growth is nonmonotonous and the phase boundaries display a rather peculiar sawtoothlike (and for large $d$ even a reentrant) behavior.

This behavior can be qualitatively understood by realizing that with increasing magnetic field the dot populated by holes will be switching to the states characterized by ever increasing positive values of the angular momentum, while the electronic dot will prefer to switch to negative angular momenta also growing in absolute value. However, since the effective mass of the holes is much larger than that of the 


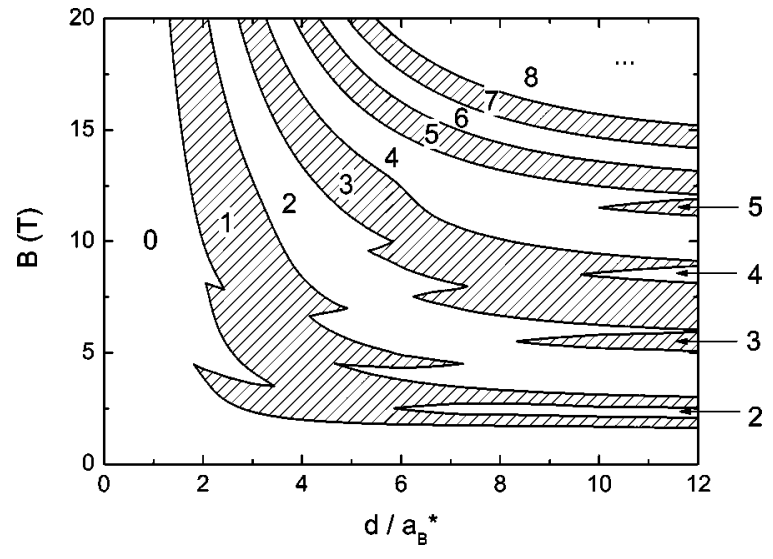

FIG. 7. The ground-state angular momentum phase diagram. The full lines denote the boundaries between the states of different angular momenta given by numbers. Areas corresponding to odd angular momenta are indicated by hash marks for clarity.

electrons, the level spacings are much smaller for the holes and thus the angular momentum transitions occur much more frequently with a growing magnetic field. Therefore, the angular momentum of the quantum dot molecule as a whole will be increasing in steps of +1 most of the time, and once in a while (whenever a transition in the electronic dot occurs) this growth will be interrupted by an occasional decrease. Similar results for double quantum dots containing 1-3 electron-hole pairs were obtained previously but only in the limit of high magnetic fields. ${ }^{22}$

For a better understanding we also consider the abovedescribed process from the point of view of a simple model. We assume that the electrons and the holes are confined in quantum dots of characteristic frequencies $\omega_{0}$ and $\Omega_{0}$, respectively, at infinitely large interdot separations. As the dots are brought closer to each other, the attractive force due to the holes creates an additional confining potential,

$$
V(r)=-\frac{2 e^{2}}{\epsilon \sqrt{r^{2}+d^{2}}} \approx-\frac{2 e^{2}}{\epsilon d}+\frac{e^{2}}{\epsilon d^{3}} r^{2}
$$

in the dot populated by the electrons. If we take this potential into account in the lowest order parabolic approximation as in Eq. (11) it can be straightforwardly summed with the bare confining potential and the potential due to the magnetic field. In this way, we can compute the effective confinement frequency as a function of the interdot distance and the magnetic field,

$$
\omega_{\mathrm{eff}}=\sqrt{\omega_{0}^{2}+\frac{1}{4} \omega_{c}^{2}+\frac{2 e^{2}}{\epsilon m^{*} d^{3}}},
$$

and determine the values of the magnetic-field strength and interdot separation at which the ground state of a two-electron system switches between the successive values of its angular momentum. ${ }^{9}$ An analogous procedure is carried out for the holes, and the results are presented in Fig. 8. The full lines denote the phase boundaries at which the angular momentum of the electrons undergoes transitions $0 \rightarrow-1 \rightarrow-2 \rightarrow \cdots$, and the dotted lines identify the analo-

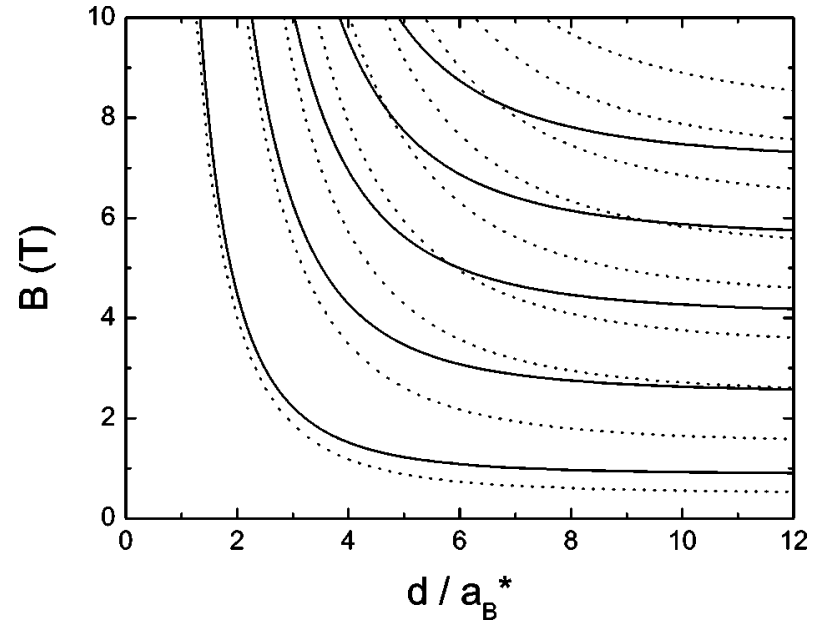

FIG. 8. The ground-state angular momentum phase diagram obtained from a simplified model. The full (dotted) lines denote the successive phase boundaries at which the angular momentum of the electron (hole) dot switches to a higher negative (positive) value.

gous phase boundaries where the angular momentum of the holes increases as $0 \rightarrow 1 \rightarrow 2 \rightarrow \cdots$. We see that the switching of the holes occur more frequently, and moreover, the phase boundaries of the holes rise more steeply in the direction of smaller interdot distances. As a result, the phase diagram in Fig. 8 contains a number of crossings of full and dotted lines as schematically shown in Fig. 9(a). The total angular momentum of all four particles is the same $(L)$ in the regions above and below the crossing point, and jumps by 2 (i.e., $L-1 \rightarrow L+1)$ when moving from the region on the left to the region on the right at the crossing point.

The inclusion of interparticle interactions at a more sophisticated level than that employed in the above simple model leads to the opening of finite gaps separating the regions of angular momenta $L-1$ and $L+1$ as shown in Fig. 9(b), and the regions corresponding to the angular momentum $L$ become connected. These modifications transform the phase boundary crossings in Fig. 8 into the sharp sawtoothshaped corners resembling the numerically calculated phase diagram in Fig. 7.

Let us now consider the effects of the Zeeman interaction. Figure 10 shows the $B$ - $d$ phase diagram corresponding to this
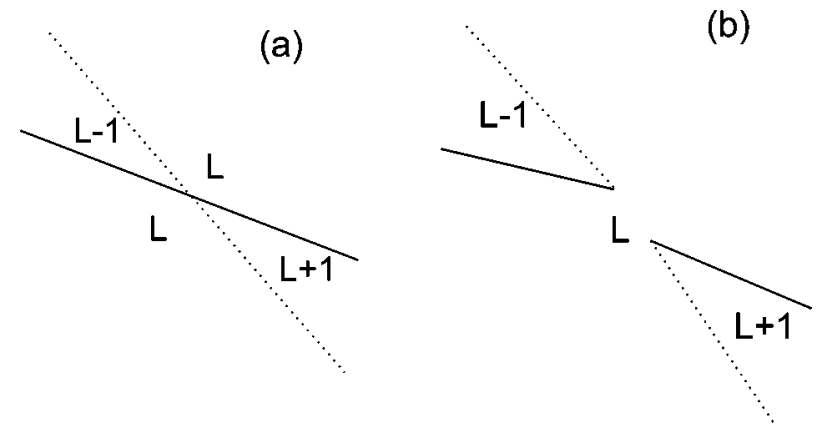

FIG. 9. The pattern of the angular momenta at the intersection of electron and hole phase boundaries. (a) shows the independent dot result, which is modified to that schematically shown in (b) by a more careful inclusion of interparticle interactions. 


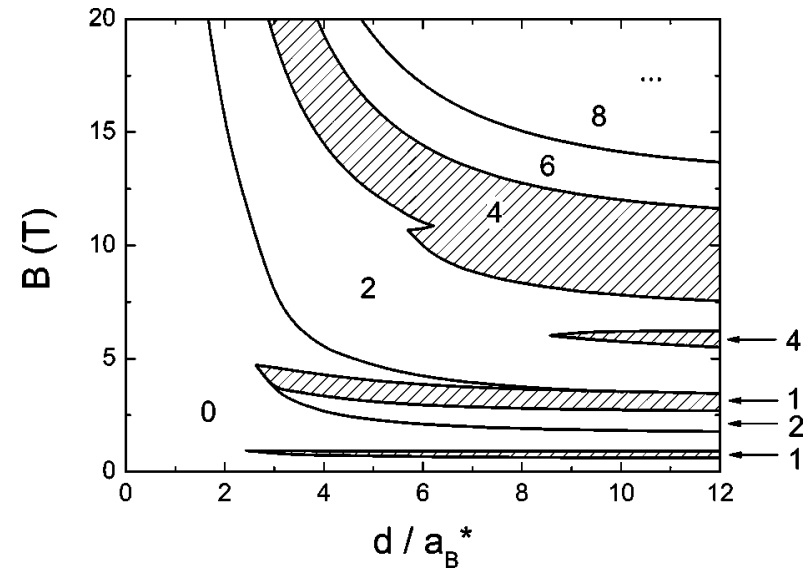

FIG. 10. The same as in Fig. 7 but now with the Zeeman interaction included. Regions corresponding to the angular momenta $L=1$ and $L=4$ are distinguished by hash marks.

case. We see that at high magnetic fields the regions corresponding to the odd values of the total angular momenta disappear. This happens because the states of even angular momenta have spin configurations of 33, i.e., with both electrons and holes polarized. Therefore, the Zeeman interaction maximally lowers the energy of these states and drives the odd angular momentum states-that have only one type of particle polarized - out of competition. The states of odd angular momentum are still present only in the low-magneticfield part of the phase diagram. Namely, we see two regions of $L=1$. The upper of the two is actually a fragment of the $L=1$ region present in the no-Zeeman-interaction case (Fig. 7). In contrast, the lower region of $L=1$ is induced by the Zeeman interaction that lowered the energy of this state below the competing $L=0$ state. Thus, we conclude that the Zeeman term brings certain rearrangements into the biexciton phase diagram, however, the characteristic sawtoothshaped phase boundaries partially survive.

\section{DIAMAGNETIC SHIFT}

Let us discuss the diamagnetic shift of the biexciton ground state. This quantity may be controlled experimentally and it clearly manifests the effects of the formation of the strongly interacting electron-hole complexes. At low magnetic fields the magnetic-field dependence of the biexciton energy can be expressed as

$$
E\left(X_{2}, B\right)=E\left(X_{2}, 0\right)+\beta B^{2},
$$

where the quantity $\beta$ is the diamagnetic shift. In Fig. 11 we show the dependence of the diamagnetic shift on the distance between the dots alongside with the biexciton binding energy at zero magnetic field. The ground state at low magnetic fields is a double singlet, therefore, these results are independent of the Zeeman term. It is interesting to observe that at small interdot distances, in the range somewhat exceeding that where bound biexcitons are formed, the diamagnetic shift becomes negative. That is, the energy of a bound biexciton decreases when a magnetic field is applied. This peculiar behavior may be understood by recalling that at close

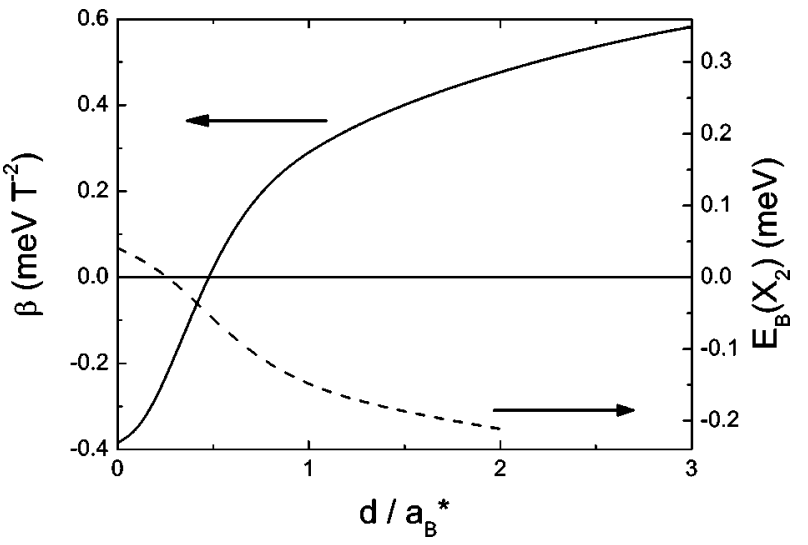

FIG. 11. The diamagnetic shift (full line, left axis) and the zeromagnetic-field binding energy (dashed line, right axis) of a biexciton confined in a double quantum dot. At low interdot distances the biexciton is bound and the diamagnetic shift becomes negative.

interdot distances the strong coupling between the dots leads to indeterminacy of angular momenta of the individual dots (see Fig. 3). Thus, while the total angular momentum of the system is zero, the electron and hole dots are in a superposition of states of various angular momenta, both $m=0$ and $m \neq 0$. This partial occupation of orbitals of negative (positive) angular momentum by the electrons (holes) leads to a negative energy dispersion with respect to the applied magnetic field.

\section{CONCLUSION}

In conclusion, we investigated a system consisting of two electrons and two holes in vertically coupled quantum dots. Interesting singlet-triplet and angular momentum transitions as a function of the magnetic field and/or interdot distance were found. At low magnetic fields and small interdot distances the ground state of the biexciton has angular momentum $L=0$ but the spin multiplicities of electron and hole subsystems simultaneously switch from a double singlet to a double triplet configuration. At larger interdot separations and stronger magnetic fields we observe ground-state angular momentum transitions with phase boundaries in the $B-d$ plane that display a sawtoothlike behavior. As a consequence, the dependence of the ground-state angular momentum on the applied magnetic field for a fixed interdot distance is nonmonotonic. We also identified the parameter range where the biexcitons are bound (unbound) with respect to dissociation into two excitons and showed that in this regime the diamagnetic shift is positive (negative).

\section{ACKNOWLEDGMENTS}

This work is supported by the European Commission GROWTH program NANOMAT project under Contract No. GSRD-CT-2001-00545, EU network of excellence SANDIE, the Belgian Interuniversity Attraction Poles (IUAP), and the Flemish Concerted Action (GOA) programmes. E.A. is supported by EU under Contract No. HPMF-CT-2001-01195. 
*Electronic address: egidijus@uia.ua.ac.be

†Electronic address: peeters@uia.ua.ac.be

${ }^{1}$ P. A. Maksym, H. Imamura, G. P. Mallon, and H. Aoki, J. Phys.: Condens. Matter 12, R299 (2000).

${ }^{2}$ L. P. Kouwenhoven, D. G. Austing, and S. Tarucha, Rep. Prog. Phys. 64, 701 (2001).

${ }^{3}$ S. M. Reimann and M. Manninen, Rev. Mod. Phys. 74, 1283 (2002).

${ }^{4}$ L. Jacak, P. Hawrylak, and A. Wójs, Quantum Dots (Springer, Berlin, 1998).

${ }^{5}$ P. A. Maksym and T. Chakraborty, Phys. Rev. B 45, 1947 (1992).

${ }^{6}$ M. Wagner, U. Merkt, and A. V. Chaplik, Phys. Rev. B 45, 1951 (1992).

${ }^{7}$ S. A. Mikhailov and N. A. Savostianova, Phys. Rev. B 66, 033307 (2002).

${ }^{8}$ M. B. Tavernier, E. Anisimovas, F. M. Peeters, B. Szafran, J. Adamowski, and S. Bednarek, Phys. Rev. B 68, 205305 (2003).

${ }^{9}$ E. Anisimovas, A. Matulis, M. B. Tavernier, and F. M. Peeters, Phys. Rev. B 69, 075305 (2004).

${ }^{10}$ T. H. Oosterkamp, J. W. Janssen, L. P. Kouwenhoven, D. G. Austing, T. Honda, and S. Tarucha, Phys. Rev. Lett. 82, 2931 (1999).
${ }^{11}$ P. A. Maksym and T. Chakraborty, Phys. Rev. Lett. 65, 108 (1990).

${ }^{12}$ F. M. Peeters and J. E. Golub, Phys. Rev. B 43, 5159 (1991).

${ }^{13}$ A. V. Filinov, M. Bonitz, and Yu. E. Lozovik, J. Phys. A 36, 5899 (2003).

${ }^{14}$ E. Anisimovas and F. M. Peeters, Phys. Rev. B 66, 075311 (2002).

${ }^{15}$ M. Rontani, F. Troiani, U. Hohenester, and E. Molinari, Solid State Commun. 119, 309 (2001).

${ }^{16}$ F. Troiani, U. Hohenester, and E. Molinari, Phys. Rev. B 65, 161301 (2002).

${ }^{17}$ Yu. E. Lozovik and V. I. Yudson, Solid State Commun. 19, 391 (1976).

${ }^{18}$ X. Zhu and P. B. Littlewood, Phys. Rev. Lett. 74, 1633 (1995).

${ }^{19}$ Yu. E. Lozovik and O. L. Berman, Phys. Scr. 55, 491 (1997).

${ }^{20}$ E. Anisimovas and F. M. Peeters, Phys. Rev. B 68, 115310 (2003).

${ }^{21}$ O. Gywat, G. Burkard, and D. Loss, Phys. Rev. B 65, 205329 (2002).

${ }^{22}$ E. Anisimovas and F. M. Peeters, Phys. Rev. B 65, 233302 (2002). 